\title{
Admission dental examination: protocol and its importance in the diagnosis of oral pathology
}

\author{
Mário Marques Fernandes ${ }^{1,2}$, Mara Rosângeles de Oliveira ${ }^{2}$, Rafael Bender Carpena de Menezes de Oliveira ${ }^{2,3}$, \\ Talita Lima de Castro, ${ }^{4,5}$, Luiz Renato Paranhos ${ }^{6}$, Eduado Daruge Júnior ${ }^{4}$
}

'Department of Community Dentistry, Faculty of Dentistry, São Paulo University, São Paulo, SP, Brazil

${ }^{2}$ Biomedical Service of Public Prosecution of Rio Grande do Sul State, RS, Brazil

${ }^{3}$ Oral and Maxillofacial Surgery Service of Irmandade Santa Casa de Misericórdia, Porto Alegre, RS, Brazil ${ }^{4}$ Department of Community Dentistry, Piracicaba Dental School, University of Campinas, Piracicaba, SP, Brazil

${ }^{5}$ Forensic Dentist, Civil Police of Rondônia State, RO, Brazil

${ }^{6}$ Oral Biology, Sacred Heart University, Bauru, SP, Brazil

\begin{abstract}
The aim of this paper is to describe an efficient clinical protocol used in admittance dental examinations, highlighting the importance of this evaluation in the diagnosis of oral pathologies. Two admission case reports in which gingival abscess and radiopaque apical lesion were found during the examination are presented for illustration purposes. A clinical examination in the admission inspection is the main factor for a good selection of treatment and prevention of future difficulties. The admission dental examination by means of a standardized clinical approach including clinical interview, physical examination and complementary exams, avoids the person to be admitted to the public service while having some pathology that might compromises his/her capacity of handling the selected function, preventing absenteeism for dental reasons and consequently a cost to the state and functional repercussion to the server.
\end{abstract}

Keywords: occupational health, occupational dentistry, routine diagnostic tests, diagnosis, oral, absenteeism.

\section{Introduction}

According to the Law 5081/66 ${ }^{1}$, which regulates the professional practice of dentistry in Brazil, the dental examination is a prerogative of the dentist. This examination also finds support in the articles 63 and 64 of the CFO Resolution $63 / 2005^{2}$, which comprise the technical expertise in administrative and labor courts as functions of the specialist in forensic dentistry, and in articles 67 and 68, the dental examinations for labor ends are competence of the specialist in Occupational Dentistry.

As part of the admission examinations, it is important not only to diagnose oral problems that affect or may affect directly the examinees, specifically investigating the epidemiology and pathology of these problems, but also to study the impact that may result in the individuals' quality of life. Such diseases can affect the work routine of an individual, causing physical and emotional damages as well as decrease their productive capacity. It must also be considered the workers' unawareness of oral health problems, the health professionals' lack of interest and knowledge regarding the study and correct interpretation of oral health 
problems that affect workers, and the need for a firm and efficient worker's health policy33.

The admission health examinations performed and the exams required from candidates for military public positions are published in the selection announcement of their contest that describes the routines and stomatognathic disabling criteria used ${ }^{4-5}$. The candidate's physical and mental health is a requirement to join the public service ${ }^{6}$, and the entrance is preceded by the performance of health inspection conducted by the official organ of expertise. Patients with diseases such as active tuberculosis, cancer, leprosy, Parkinson's disease, ankylosing spondyloarthrosis, later stages of Paget's Disease (osteitis deformans), acquired immunodeficiency syndrome (AIDS), among others, may enter the public service provided that the expert entry assessment and the probation period, present ability to exercise the function, demonstrate clinical attendance and adherence to appropriate treatment in scientific indication standards approved by health authorities ${ }^{7}$.

Considering the possibility of clinical findings with systemic origin in addition to the specific conditions of oral health in the admission examinations carried out, there are several studies that support the importance of knowledge of oral manifestations of diseases such as tuberculosis ${ }^{8}$, leprosy ${ }^{9}$, Parkinson's disease ${ }^{10}$, ankylosing spondyloarthrosis ${ }^{11}$, later stages of Paget's disease ${ }^{12}$ and AIDS $^{8,13}$.

This article aims to describe an efficient protocol used in admittance dental examinations, highlighting the importance of this evaluation in the diagnosis of oral pathologies. Two admission case reports are presented for illustration purposes.

\section{Protocol}

The study was approved by the Research Ethics Committee of the Piracicaba Dental School, University of São Paulo, Brazil (protocol number 144/2008).

In reviewing the literature on the subject, using the descriptors of this study and related, little was found published in indexed journals. The recommended routines to perform the dental examination for entrance into public service were similar to those used in clinical management.

When presenting the cases of a service of occupational medicine in a large hospital in Sao Paulo, Santos et al. ${ }^{14}$ affirmed that the pre-admission examination requires a rigorous assessment of the conditions of oral infection, aesthetics and other diseases that originate in the mouth and may have systemic repercussions. The authors highlighted that the criteria adopted in oral evaluation and to release the candidate for performing his/her functions can interfere with the amount of emergency care and the number of later removals ${ }^{8,14}$.

The protocol presented here was developed by dentists based on clinical experience and scientific literature for use in admission dental evaluations in public institutions at a first moment, with possibility of being extended to the private sphere. Considering the expert nature of the service, the protocol brings elements that help in the description and characterization of the individuals and the morbid events that they can present during the examination. These elements may be useful in future evaluations as identification, redeployment functional exams, removal, granting permission for health care, etc.

As in all clinical investigations to establish a diagnosis, the protocol involves anamnesis, physical examination and complementary tests. The anamnesis is obtained mainly through the filling of the Term of Declaration of Health for the Purpose of Entry, an instrument used by the service staff for the entrant answer questions on general health and thus give his/her views on issues related to current and past illnesses, medication use, alcohol use and use of illegal drugs. The term must be dated and signed by the entrant, who is responsible for the accuracy of the information provided. In the case of inaccuracy or omission of any of the questions raised, the corresponding admission report can be canceled.

On the day of expertise, as complementary exams, the following radiographic examinations must be done: a panoramic radiograph, for evaluation of the bone arcades, adjacent structures and possible pathological changes, and bitewing radiographs of bilateral molars and premolars, aiming at the evaluation of caries, marginal adaptation of restorations and the alveolar bone crest. It is oriented that the radiographs are recent dated up to 3 months prior to the expertise. Imaging or biochemical tests may also be required as well as reports of various specialties for information and clarification as necessary, through formal acquiescence of the patient ${ }^{15}$. Pregnant women, regardless of gestational age, are exempted from submission of radiographs because of the risks of the procedure, although there is controversy about this subject in literature.

On physical examination, it is evaluated general extraoral characteristics such as facial anthropometry (if the examinee has facial skeletal pattern as dolichofacial, brachyfacial or mesofacial type), the temporomandibular joint (if there is clicking, crepitation, limitation of mouth opening, lateral deviation or pain), and the intraoral characteristics such as occlusion, the relationship between the dental arches, transverse, anteroposterior and molar relation, presence of oral parafunction and passive lib seal, stomatological evaluation, observing the lips, tongue, mouth floor, vestibule, palate and oral mucosa, in addition to accurate intraoral inspection of the teeth and periodontal evaluation, and the execution of the odontogram. Table 1 illustrates the protocol proposed and Table 2 contains the basic materials required.

It is worth noting the importance of assessing the immunity of employees of hospitals and insalubrious centers. The collection of oral fluids as a source of material for noninvasive diagnostic tests should be performed by a dental professional as a routine procedure during pre-admissional physical examination ${ }^{14}$.

The entrants who present, at the time of expertise, conditions that compromise the ability to exercise the function must be referred for treatment before the tenure ceremony, and must be submitted to a new assessment after the procedure. In these situations, it is delivered to the examinees the Technical Report Routing, which describes 
Table 1: Clinical protocol suggested for admission dental examination

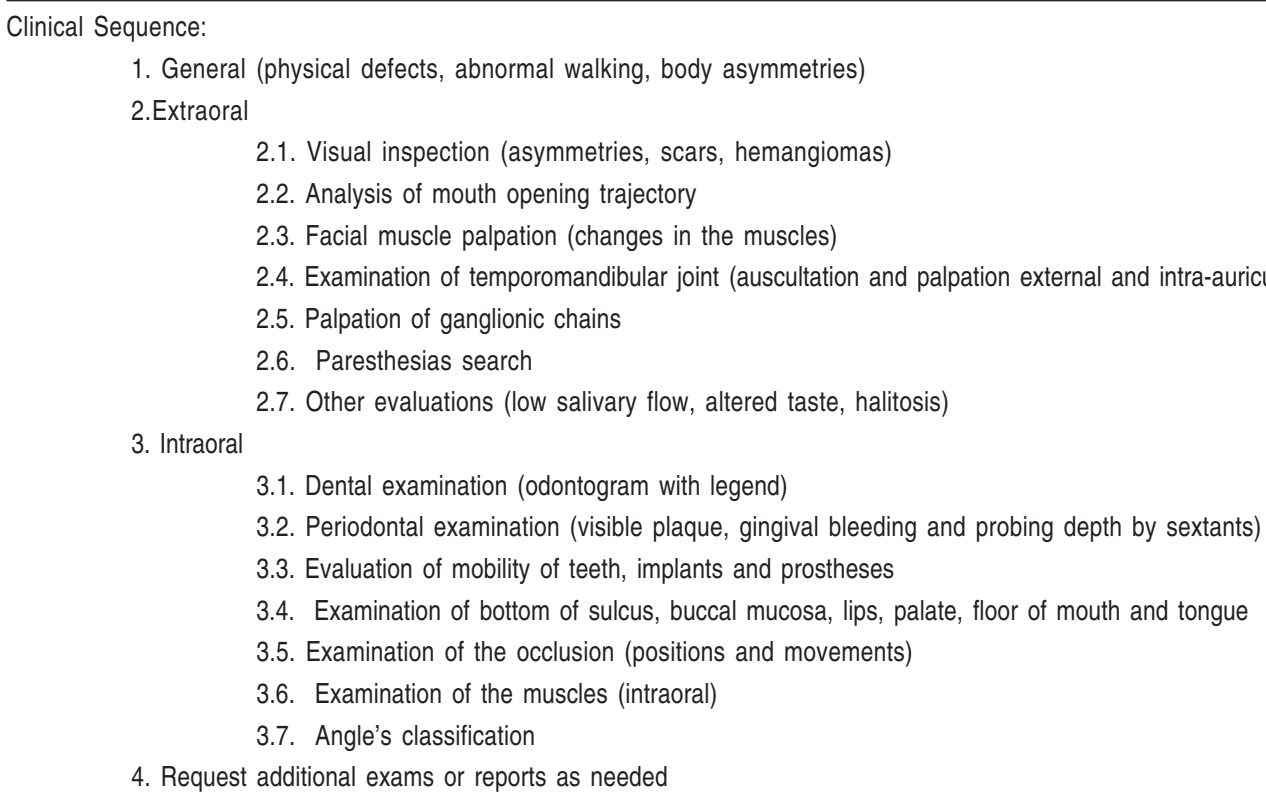

Table 2: List of basic instruments and materials suggested for the examinations

Materials and instruments for the admission dental examination:
1. Personal protective equipment (Apron, gloves, mask, glasses and bonnets)
2. Dental probe explorer No 5 , mirror and clinical tweezers
3. Periodontal probe
4. Articulating paper tweezer (Müller tweezer)
5. Articulating paper
6. Tongue depressor sticks
7. Dental Floss
8. Dental aspirator, cotton rolls, gauze
9. Plaque disclosing solution
10. Lip retractors
11. Stethoscope
12. Camera / Camcorder
13. Lightbox and magnifying glass
14. Spray for pulp sensitivity testing

the pathology, requests the appropriate treatment and emphasizes the need to return for final evaluation. When the deadlines for tenure are scarce, the expert dentist can declare the newcomer temporarily unfit, and then the newcomer has up to 30 days for new examination and subsequent act of tenure on the job ${ }^{7}$. All findings from the clinical examination are recorded in the newcomer's medical enchiridion, as well as additional exams and specialty reports. The newcomers who present any clinical changes that do not compromise their occupational ability are qualified and classified as "capable but with dental needs". The dentist must issue a conclusive report regarding the ability or inability to work, support technically this decision, and also guide the examinee on the need for treatment, when the candidate is not performing ${ }^{15}$.

On this basis, the pre-admission physical examination is of key importance, which, besides its main function of analyzing the individual's labor capacity, it still plays the role of detecting and diagnosing oral disease and advising the newcomer about the most suitable treatment.

\section{Case Report 1: Alterations in the maxilla}

The candidate was a 38-year-old Caucasian male with history of extraction of the maxillary canines nearly 20 years before the admittance dental examination. He mentioned drug therapy, 2 months ago, due to a gingival abscess between the maxillary right lateral incisor and first premolar. Clinical examination of the vestibule revealed the presence of mild edema and hyperemia in the gingival mucosa located between the teeth 12 and 14. On the occasion of the expert's examination, an attest issued by the assistant dentist was delivered, proving to be the newcomer in dental treatment for the pathology found. The periapical radiographs showed divergent roots in the region between teeth 12 and 14 (Figure 1), and between teeth 22 and 24 (Figure 2), with a radiopaque area surrounded by a less radiopaque halo bilateral.

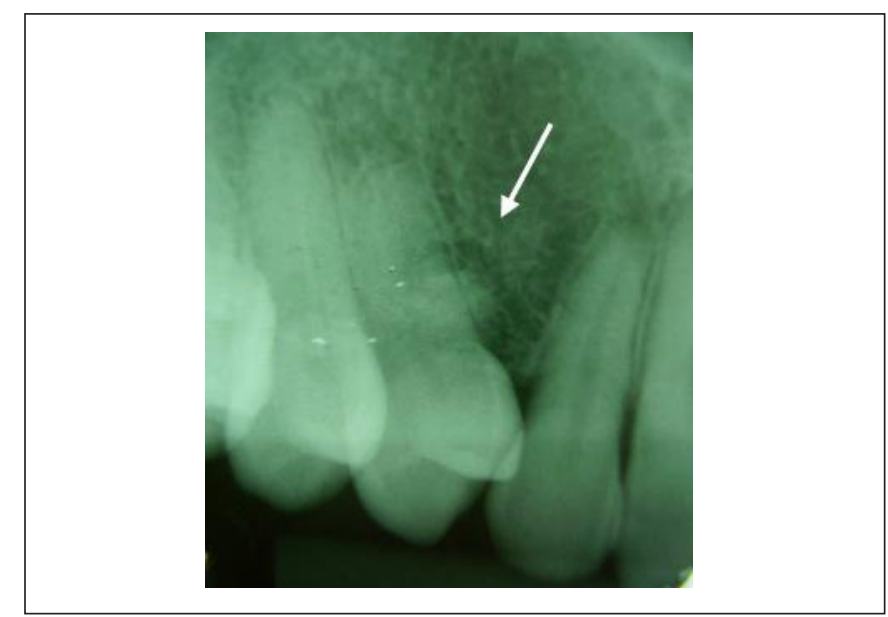

Fig. 1 - Radiopaque lesion in the maxilla - Periapical radiographic image in the right side. 


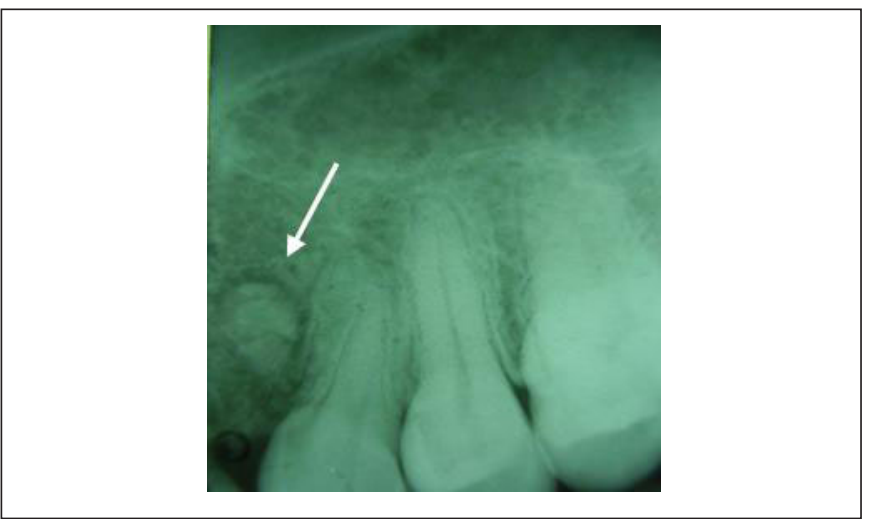

Fig. 2 - Radiopaque lesion in the maxilla - Periapical radiographic image in the left side.

\section{Case Report 2: Alterations in the mandible}

A 32-year-old Caucasian male presented for dental expertise with the radiograph examinations requested. The analysis of the panoramic radiograph (Figure 3) revealed diffuse radiopaque area in the root region of tooth 46 . Palpation of the vestibule (as oriented in protocol) showed no volume increase in the region, and the pulp sensitivity test was positive. The newcomer reported having knowledge of the injury for 10 years since the time he was an undergraduate dental student, and he believed that the lesion had increased a little. New periapical and occlusal radiographs were requested for a more accurate evaluation. The radiologist's report indicated radiopaque image of dense aspect associated with the mesial root of tooth 46, extending apically and to the inter-root space, with obscuration of the periodontal ligament space and lamina dura of apical third of the mesial root presenting irregular form and no marginal radiolucent line (Figure 4). The occlusal radiograph did not show changes in vestibular or lingual cortical (Figure 5). The radiologist's report suggested pathology compatible with idiopathic osteosclerosis.

\section{Discussion}

Given the issues mentioned above, the physical examination before employment is of great relevance, which,

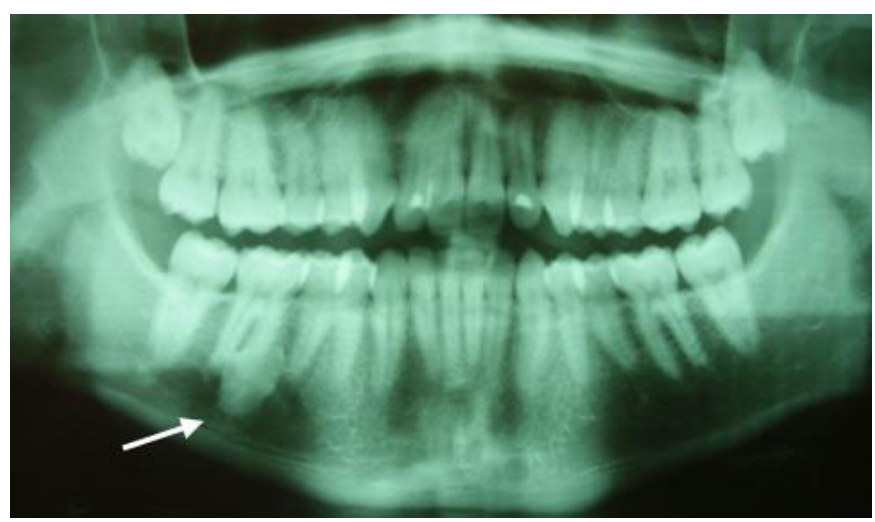

Fig. 3 - Dense radiopaque lesion in the mandible - Panoramic radiographic image.

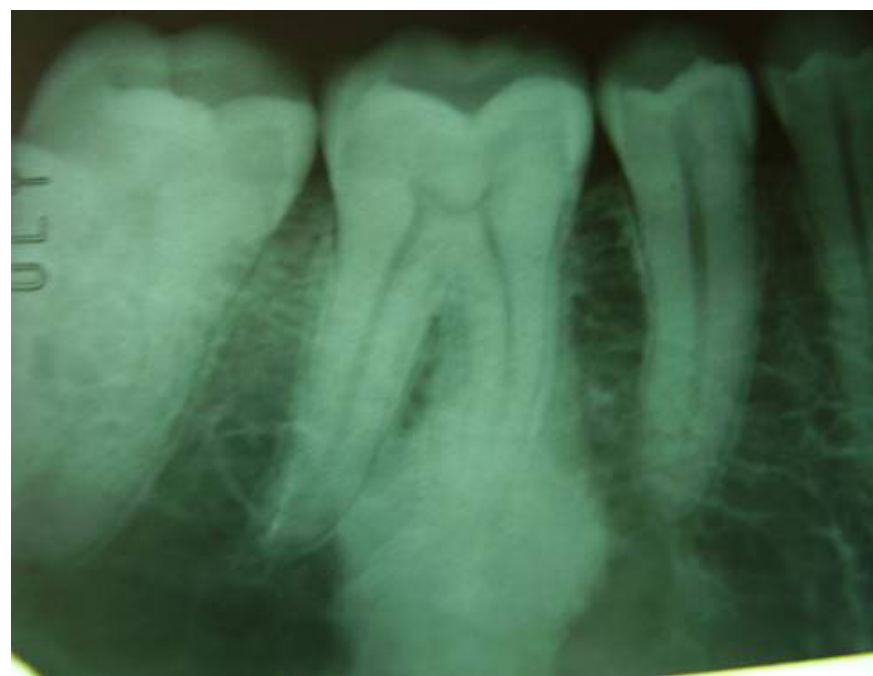

Fig. 4 - Radiopaque lesion associated with the mesial root of tooth 46 , extending apically to inter-root space - Periapical radiographic image.

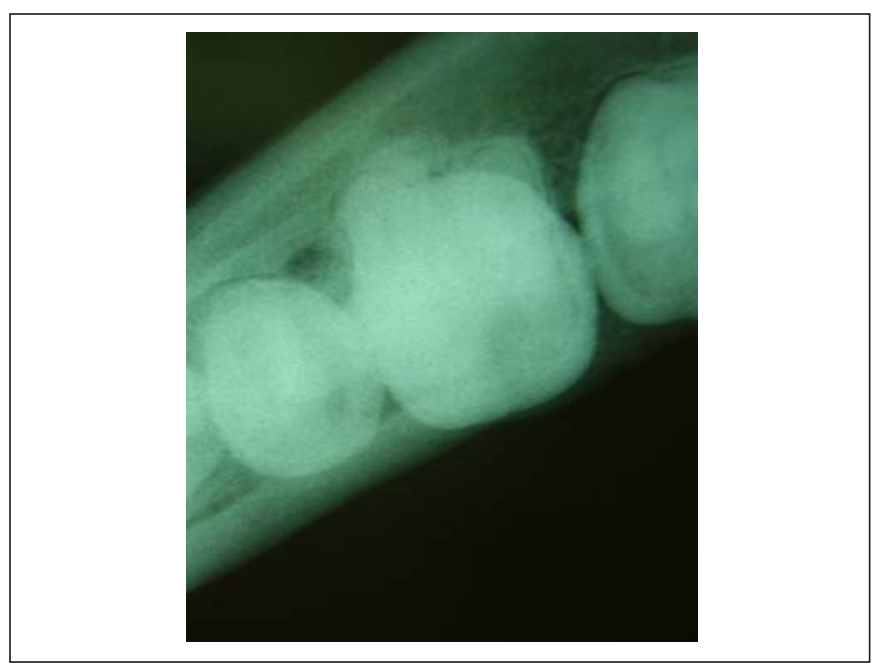

Fig. 5 - Lateralized occlusal radiographic image, without changes in vestibular or lingual cortical.

besides its main function to analyze the work capacity of the individual, still plays the role of detecting and diagnosing oral diseases, sending the entrant to appropriate treatment.

In Case 1, calcifying odontogenic cyst (Gorlin's cyst) in one of its variants and odontoma ${ }^{16}$ were possible differential diagnoses for the lesion. A review of the current classification of odontogenic tumors showed that odontomas are considered by some as developmental disorders and not neoplasias ${ }^{17}$. Considering that the newcomer's activities did not include continuous exposure to any risk agent (biological, chemical or physical), and knowing about the injury, its treatment and prognosis, the expert opted for approving the applicant's ability. The examinee was oriented to continue with clinical follow-up with the dental specialist.

In Case 2, a possible diagnosis for the lesion included condensing osteitis, focal sclerosing osteomyelitis, periradicular osteosclerosis, sclerosing osteitis and sclerotic bone $^{18}$. As similar changes to that related to the pathology, are described sclerosing osteitis and benign cementoblastoma, 
the latter being a rare case, which is evident before the age of 25 , and the most affected tooth is the mandibular first molar, with pulp vitality, with slow growth and may expand bone corticals ${ }^{16}$. Radiographically the tumor has adhered to the tooth and appears as a dense radiopaque mass well circumscribed surrounded by a thin uniform radiolucent halo, and the root outline is usually unseen. It is worthwhile making the differential diagnosis of odontoma, osteoblastoma, hypercementosis and focal sclerosing osteitis ${ }^{19}$. Given this context, the radiopaque radiographic finding, the clinical characteristics at the time of examination (noting the absence of signs and symptoms), the prognosis of the injury, and even if the newcomer's duties comprised in the public office were not about oral or physical effort or planned exposure to any agent of risk, the dental expert opted to classify the candidate as being capable, believing that the labor ability of the examinee at the time of examination was not hampered by the lesion. The examinee was oriented to maintain clinical and radiographic follow-up with the assistant dentist. Both patients agreed to the publication of their cases by signing an informed consent form.

It is highlighted here the importance of a detailed and carefully done pre-admission examination, following the standards and protocols, in order to detect possible oral alterations. Oral diseases are often chronic and may cause physical and emotional distress, impaired concentration, decrease in employee productivity, or even work accidents ${ }^{14}$. These disorders are entirely avoidable if the examiners are aware during the clinical examination, about the function of realizing and diagnosing the presented oral pathologies.

An excellent clinical examination in the admission inspection is a major factor of a good selection and prevention of future difficulties. It extends and maintains the operational training of selected personnel, prevents loss of financial and human resources in the recovery of easily identifiable deficiencies, and is of paramount importance in the prevention of future disabling causes in the worker career ${ }^{20}$.

The dentist, being an official expert, performs single expertise, hospital and domiciled exams, in administrative processes and procedures, in order to assess the work condition of the examinee in circumstances that are within field of dentistry. The dentist must act impartiality and neutrality, not admitting any kind of pressure, imposition, constraint or restriction, and may even refuse to continue the examination if that happens ${ }^{15}$. There are few public institutions that have a dentist working in sectors that perform admittance examinations or related to occupational issues exams, but it is noted that the Brazilian law has evolved to regulate this practice ${ }^{21}$.

On this matter, it is emphasized the presence of dentists in the staffs of Public Ministry of the state of Rio Grande do Sul, located in the "Biomedical Services", the official organ of expertise of that institution. This department is responsible for the entrance exams, granting of licenses, and other removals provided in law ${ }^{6}$. The tasks of dentist's office are listed as, among others, carrying out inspections within dentistry, signing attests and reports for license granting, in addition to performing or assisting expertise, including the admission or entrance examinations ${ }^{22}$.

Considering the admittance examinations for entry into public service, the expert who conducts the assessment shall keep in mind that the concept of physical ability is linked to individual's capability to develop his work activities, included in the tasks relevant to the position for which he/she is being admitted at that time. The evaluator must also list the diseases encountered and the possible need for withdrawal to treatment, both aesthetic and functional, thus avoiding damage to work after the candidate's entry. In the dental field, the most common needs of treatment found in the admittance examinations performed by the dental service are linked to the signs and symptoms of dental cavities (including in this context its evolution for periapical pathologies) and periodontal problems, as well as any pathology related to cysts and tumors, as shown in the case reports presented in this paper.

The studies found in literature about the dentist's work in occupational activities are scarce and the dental criteria used for fitness in admission are varied ${ }^{4-5}$. According to the survey, there are few places that perform the dental entrance exam in public and private areas, for different reasons ${ }^{3}$. Studies on the importance of information obtained from the admissions exams reinforce the idea that the inclusion of dentistry in the Medical Control Program for Occupational Health, normalized as $\mathrm{PCMSO}^{23}$, is important to attest to oral health within the system of occupational health, and to create a database for the dental field. Consequently, it is seems clear the importance of the collaboration of dentistry with medicine in the production of admission and periodic medical examinations (occupational), being inserted, together with the various public and private sectors, in the concept of life quality at the workplace ${ }^{3}$. Therefore, the admission dental exam prevents the person from entering the office with any condition that can lead to problems at the workplace and even to removals, with expenses to the state and functional consequences to the server.

\section{References}

1. Brazil. Law n. $-5.081,1996$ August 24. Available from: http:// www.planalto.gov.br/ccivil_03/Leis/L5081.htm.

2. Federal Council of Dentistry. Resolution n. ${ }^{\circ}$ 63/2005, 2005 April 19. Available from: http://cfo.org.br/servicos-e-consultas/ato-normativo/ ?id=986.

3. Araujo ME, Gonini Jr A. Worker Oral Health: The Admissional and Periodical Examinations as an Information Health System. Odontol Soc. 1999; 1: 15-8.

4. Brazil. Ministry of Defense. Air Force Command. Health Direction. Bulletin n. $-200,2006$ October 27.

5. Brazil. Ministry of Defense. Navy Command. Education Direction. Announcement of the Selection Process for entry into the Marine Health Corps, Annex V, 2007 December 30.

6. Brazil. Rio Grande do Sul State. Complementary Law n. $.010 .098,1994$ February 03. Available from: http: //www.al.rs.gov.br/Legis/normas/ 10098.pdf.

7. Brazil. Rio Grande do Sul State. Complementary Law n. ${ }^{\circ} 11.836,2002$ October 21. Available from: http: //www.al.rs.gov.br/Legis/Arquivos/ 11.836.pdf. 
8. Petruzzi MNMR, Salum FG, Cherubini K, Figueiredo MAZ. Epidemiological characteristics and HIV-related oral lesions observed in patients from a Southern Brazilian city. Rev Odonto Cienc. 2012; 27: 115-20.

9. Ghosh S, Gadda RB, Vengal M, Pai KM, Balachandran C, Rao R et al. Oro-facial aspects of leprosy: Report of two cases with literature review. Med Oral Patol Oral Cir Bucal 2010; 15: E459-62.

10. DeBowes SL, Tolle SL, Bruhn AM. Parkinson's disease: considerations for dental hygienists. Int J Dent Hyg. 2012; 11: 15-21.

11. Arakeri G, Kusanale A, Zaki GA, Brennan PA. Pathogenesis of posttraumatic ankylosis of the temporomandibular joint: a critical review. $\mathrm{Br} \mathrm{J}$ Oral Maxillofac Surg. 2012; 50: 8-12.

12. Garg K, Devi P, Thimmarasa VB, Mehrotra V. Osteomyelitis of the mandible as a complicating factor in paget's disease: Acase report. Indian J Dent. 2012; 3: 110-7.

13. Girardi FM, Scroferneker ML, Gava V, Pruinelli R. Head and Neck Manifestations of Paracoccidioidomycosis: An Epidemiological Study of 36 Cases in Brazil. Mycopathologia. 2012; 173: 139-44.

14. Santos PSS, Pinto MF, Guimarães Neto JA. Occupational Dentistry in the hospital setting. Rev Odonto Cienc. 2008; 23: 307-10.

15. Federal Council of Dentistry. Resolution n. - 87/2009. Available from: http://cfo.org.br/servicos-e-consultas/ato-normativo/?id=1356.

16. Bishop JA. Atlas of Oral and Maxillofacial Histopathology. Am J Surg Pathol. 2013; 37: 152.

17. Costa DO, Maurício AS, Faria PA, Silva LE, Mosqueda-Taylor A, Lourenço SQ. Odontogenic tumors: a retrospective study of four Brazilian diagnostic pathology centers. Med Oral Pathol Oral Cir Bucal. 2012; 17: e389-94.

18. Capelas J A, Albuquerque B, Paulo S, Ferreira MM, Ginjeira A. Glossary of Endodontic Terms.- Part II. Rev Port Estomatol Cir Maxilofac. 2008; 49: 57-62.

19. Neelakandan RS, Deshpande A, Krithika C, Bhargava D. Maxillary cementoblastoma - a rarity. Oral Maxillofac Surg. 2012; 16: 119-21.

20. Velloso LFN, Ferreira LACN, Perro Filho MJT, Caiafa JS. Health investigation for admittance to active navy service: importance of systematized clinical examination and repercussion at short, medium and long term of selection. Arq Bras Med Nav. 1989; 50: 151-60.

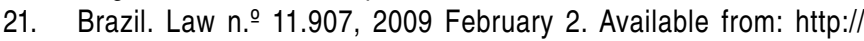
www.planalto.gov.br/ccivil_03/_ato2007-2010/2009/Lei/L11907.htm.

22. Brazil. Rio Grande do Sul State. Law n. .0 10.559, 1995 October 19. Available from: http://www.al.rs.gov.br/LegisComp/Arquivos/ Lei\%20n\%C2\%BA\%2010.559.pdf.

23. Brazil. Ministry of Labour and Employment - Department of Safety and Health at Work. Regulatory Standard 7 (NR 7), 1978 July 06. Available from: http://www.mte.gov.br/legislacao/normas_regulamentadoras/ nr_07_at.pdf. 